\title{
Fully nonlinear curvature flow of axially symmetric hypersurfaces
}

\author{
James A. McCoy, Fatemah Y. Y. Mofarreh and \\ Valentina-Mira Wheeler
}

\begin{abstract}
Recently, fully nonlinear curvature flow of a certain class of axially symmetric hypersurfaces with boundary conditions time of existence was obtained, in the case of convex speeds (J. A. McCoy et al., Annali di Matematica Pura ed Applicata 1-13, 2013). In this paper we remove the convexity condition on the speed in the case it is homogeneous of degree one in the principal curvatures and the boundary conditions are pure Neumann. Moreover, we classify the singularities of the flow of a larger class of axially symmetric hypersurfaces as Type I. Our approach to remove the convexity requirement on the speed is based upon earlier work of Andrews for evolving convex surfaces (B. H. Andrews, Invent Math 138(1):151-161, 1999; Calc Var Partial Differ Equ 39(3-4):649-657, 2010); these arguments for obtaining a 'curvature pinching estimate' may be adapted to this setting due to axial symmetry. As further applications of curvature pinching in this setting, we show that closed, convex, axially symmetric hypersurfaces contract under the flow to round points, and hypersurfaces contracting self-similarly are necessarily spheres. These results are new for $n \geq 3$.
\end{abstract}

Mathematics Subject Classification. Primary 35K55, 35R35, 53C44; Secondary 35K60.

Keywords. Curvature flow, Parabolic partial differential equation, Hypersurface, Initial-boundary value problem, Neumann boundary condition.

The research of James A. McCoy and Valentina-Mira Wheeler was supported by Discovery Project DP120100097 of the Australian Research Council. The research of Fatemah Y. Y. Mofarreh was supported by a postgraduate scholarship from Princess Nora bin Abdulrahman University. 


\section{Introduction}

Recently, the first two authors, together with Williams, investigated a class of fully-nonlinear curvature flows of axially symmetry hypersurfaces with boundary conditions [18], following earlier studies of the mean curvature flow in this setting $[1,11,12,14,15]$. Critical in [18] was the convexity of the speed as a function of the principal curvatures. In this article, we remove that restriction in the case of pure Neumann boundary conditions by consideration of an appropriate pinching function of the principal curvatures of the evolving hypersurface. Our idea is based upon earlier constructions for evolving convex surfaces by Andrews [3,5] of preserved pinching functions, constructions which were also used by the first author $[16,17]$. Very recently, the first author, together with Andrews and Langford [6], controlled a pinching function under fully nonlinear curvature flow of nonconvex surfaces. The monotonicity of these curvature pinching functions is obtained via application of the maximum principle on the evolving surfaces; constructions use heavily the Codazzi equations and homogeneity of the speed and the pinching function which provide sufficient information in the case of surfaces. However, in this article, we are able to establish curvature pinching for axially symmetric hypersurfaces, that is, for $n$-dimensional hypersurfaces with $\mathbb{S}^{n-1}$ symmetry, since enough of the gradient terms disappear from the evolution equation for the pinching function to permit a similar analysis to earlier work. Preservation of a pinching ratio under the flow implies uniform parabolicity of the flow equation and bounds above and below on all symmetric functions of the principal curvatures that are homogeneous of degree zero.

As in the case of the mean curvature flow [14], axially symmetric hypersurfaces evolve under our fully nonlinear flows for a finite maximal time $T$ when a curvature singularity develops. We classify the curvature singularity as 'Type I', as in the case of mean curvature flow, however our analysis is somewhat different to that in [14] due to the fully nonlinear nature of our flow. We refer the reader to $[6,7]$ for discussions on the importance and consequences of classifying singularities of fully nonlinear curvature flow.

The structure of this article is as follows. In Sect. 2, we set the majority of our notation, which is consistent with earlier work and we detail the properties of the nonlinear speed functions under consideration. In Sect. 3 we briefly describe the geometric features of axially symmetric hypersurfaces that we will require in our analysis, in particular we provide expressions for the Christoffel symbols and the gradient of the Weingarten map in this setting. In Sect. 4 we discuss the flow problem and prove some preliminary results. The system of evolution equations describing the position vector of the evolving hypersurface is equivalent to a scalar evolution equation for the corresponding height of the graph of the generating curve above the $x$ axis. We obtain a lower bound on the rotational curvatures that is inversely proportional to the height of the evolving graph; this implies a similar bound on the axial derivative of the graph function. In Sect. 5 we prove the crucial pinching estimate, 
listing several important corollaries including a bound on the ratio of the principal curvatures. We also apply curvature pinching to remove the convexity condition in the main result of [18] in the case of pure Neumann boundary conditions and to show that the second axial derivative of the graph function is bounded inversely proportional to the square of the graph height. In Sect. 6 we characterise the maximal time as the time of blow-up of the norm of the second fundamental form and we show that in this setting, as is the case with convex surfaces $[2,5]$, the singularity is Type I. In Sect. 7 , we turn our attention to contracting closed, convex, axially symmetric hypersurfaces, showing that such hypersurfaces contract under our class of flow to asymptotically spherical points in finite time. Moreover, if the contraction is self-similar, then the hypersurface must be a sphere. For $n \geq 3$ these are a new results: they generalise to higher dimensions the results in $[5,17]$ and can also be thought of as a relaxation of requirements on the speed or the initial data of other works.

The authors would like to thank Professor Graham Williams and Dr Glen Wheeler for their interest in this work and the anonymous referee whose suggestion led to the inclusion of Sect. 7 .

\section{Preliminaries}

We will use similar notation to earlier work. In particular, $g=\left\{g_{i j}\right\}, A=\left\{h_{i j}\right\}$ and $\mathcal{W}=\left\{h_{j}^{i}\right\}$ denote respectively the metric, second fundamental form and Weingarten map of $M_{t}$. The mean curvature of $M_{t}$ is

$$
H=g^{i j} h_{i j}=h_{i}^{i}
$$

and the norm of the second fundamental form is

$$
|A|^{2}=g^{i j} g^{l m} h_{i l} h_{j m}=h_{l}^{j} h_{j}{ }^{l}
$$

where $g^{i j}$ is the $(i, j)$-entry of the inverse of the matrix $\left(g_{i j}\right)$. Throughout this paper we sum over repeated indices from 1 to $n$ unless otherwise indicated. We will denote by $\nabla$ the covariant derivative on the hypersurface and by $\bar{\nabla}$ the derivative on $[0, a] \times \mathbb{S}^{n-1}$. In particular, $\bar{\nabla}_{1}=\frac{\partial}{\partial x}$, while $\bar{\nabla}_{j}, j \geq 2$ denote the $\mathbb{S}^{n-1}$ derivatives.

Our nonlinear speed functions $F$ should have the following properties:

\section{Conditions 2.1.}

(i) $F(\mathcal{W})=f(\kappa(\mathcal{W}))$ where $\kappa(\mathcal{W})$ gives the eigenvalues of $\mathcal{W}$ and $f$ is a smooth, symmetric function defined on an open convex cone $\Gamma$ containing the positive cone

$$
\Gamma_{+}=\left\{\kappa=\left(\kappa_{1}, \ldots, \kappa_{n}\right) \in \mathbb{R}^{n}: \kappa_{i}>0 \quad \text { for all } i=1,2, \ldots, n\right\} .
$$

(ii) $f$ is strictly increasing in each argument: $\frac{\partial f}{\partial \kappa_{i}}>0$ for each $i=1, \ldots, n$ at every point of $\Gamma$.

(iii) $f$ is homogeneous of degree one: $f(k \kappa)=k f(\kappa)$ for any $k>0$.

(iv) $f$ is positive and normalised, $f(1, \ldots, 1)=1$. 
As mentioned above, of note in this article is the absence of a convexity condition on $f$. Speeds which satisfy the above conditions are discussed in $[6,17]$; in particular, here we can take linear combinations of examples regardless of individual convexity or concavity. Many speeds may be built from combinations of elementary symmetric functions of the principal curvatures.

We will denote by $\left(\dot{F}^{k l}\right)$ the matrix of first partial derivatives of $F$ with respect to the components of its argument:

$$
\left.\frac{\partial}{\partial s} F(A+s B)\right|_{s=0}=\dot{F}^{k l}(A) B_{k l}
$$

Similarly for the second partial derivatives of $F$ we write

$$
\left.\frac{\partial^{2}}{\partial s^{2}} F(A+s B)\right|_{s=0}=\ddot{F}^{k l, r s}(A) B_{k l} B_{r s} .
$$

We will also use the notation

$$
\dot{f}_{i}(\kappa)=\frac{\partial f}{\partial \kappa_{i}}(\kappa) \text { and } \ddot{f}_{i j}(\kappa)=\frac{\partial^{2} f}{\partial \kappa_{i} \kappa_{j}}(\kappa) .
$$

Unless otherwise indicated, throughout this paper we will always evaluate partial derivatives of $F$ at $\mathcal{W}$ and partial derivatives of $f$ at $\kappa(\mathcal{W})$.

In a local orthonormal frame of eigenvectors of $\mathcal{W}$, we may write $\ddot{F}$ in terms of $\ddot{f}$ and $\dot{f}$ as follows, for any symmetric matrix $B$ :

$$
\ddot{F}^{p q, r s}(\mathcal{W}) B_{p q} B_{r s}=\ddot{f}^{p r} B_{p p} B_{r r}+2 \sum_{p<r} \frac{\dot{f}^{p}(\kappa)-\dot{f}^{r}(\kappa)}{\kappa_{p}-\kappa_{r}}\left(B_{p r}\right)^{2} .
$$

This formula makes sense as a limit in the case of any repeated values of $\kappa_{i}$. For details of this, we refer the reader to [4], for example.

\section{Geometry of axially symmetric hypersurfaces}

An $n$-dimensional axially-symmetric hypersurface $M$ can be specified by a corresponding strictly positive and suitably smooth function $u:[0, a] \rightarrow \mathbb{R}$ such that $M$ is parametrised by $X:[0, a] \times \mathbb{S}^{n-1} \rightarrow \mathbb{R}^{n+1}$, where

$$
X(x, \omega)=(x, u(x) \omega) .
$$

We will assume that $u$ is smooth enough on $[0, a]$ for all derivatives we use to make sense. Throughout the paper, derivatives at the endpoints $x=0$ and $x=a$ are interpreted naturally as one-sided derivatives.

As in [14] we will set $y=\sqrt{|X|^{2}-\left\langle X, e_{1}\right\rangle^{2}}$, where $e_{1}$ is the unit vector in the $x_{1}$ axis direction and $q=\left\langle\nu, e_{1}\right\rangle y^{-1}=-y^{\prime} \kappa_{2}$, where $\nu$ is the outer unit normal to $M$. In a slight abuse of notation, we will often write $u$ in place of $y$ to emphasise the dependence of the graph function only on the axial direction $x=x_{1}$. 
It is straightforward to check that the matrices of the metric, second fundamental form and Weingarten map of $M$ are given respectively by

$$
\begin{aligned}
g_{i j}=\left(\begin{array}{cc}
1+u_{x}^{2} & 0 \\
0 & u^{2} \bar{\sigma}_{i j}
\end{array}\right), h_{i j} & =\left(\begin{array}{ll}
-\frac{u_{x x}}{\sqrt{1+u_{x}^{2}}} & 0 \\
0 & \frac{u}{\sqrt{1+u_{x}^{2}}} \bar{\sigma}_{i j}
\end{array}\right) \text { and } h_{j}^{i}{ }_{j} \\
& =\left(\begin{array}{ll}
-\frac{u_{x x}}{\left[1+u_{x}^{2}\right]^{\frac{3}{2}}} & 0 \\
0 & \frac{1}{u \sqrt{1+u_{x}^{2}}} \delta^{i}{ }_{j}
\end{array}\right),
\end{aligned}
$$

where $\bar{\sigma}_{i j}$ denotes the metric on $\mathbb{S}^{n-1}, \delta^{i}{ }_{j}$ is the Kronecker delta and we write $u_{x}=\frac{d u}{d x}$.

We will denote by $\kappa_{1}$ the curvature of the generating curve $(x, u(x))$ of the surface of revolution, that is, $\kappa_{1}$ is the 'axial curvature'. We denote by $\kappa_{2}$ the 'rotational curvatures', $\kappa_{2}=\kappa_{3}=\ldots=\kappa_{n}$. In view of the above expression for the matrix of the Weingarten map, the principal curvatures of $M$ are clearly given by

$$
\kappa_{1}=\frac{-u_{x x}}{\left[1+u_{x}^{2}\right]^{\frac{3}{2}}}, \quad \kappa_{j}=\frac{1}{u \sqrt{1+u_{x}^{2}}}, \quad j=2, \ldots, n
$$

Because of the axial symmetry, many of the derivatives of the second fundamental form for axially symmetric surfaces are identically equal to zero. We compute them explicitly, via the Christoffel symbols.

Lemma 3.1. In normal coordinates at any particular point, the only nonzero Christoffel symbols of the induced metric of axially symmetric hypersurfaces of the form (2) are

$$
\Gamma_{11}^{1}=\frac{u_{x} u_{x x}}{1+u_{x}^{2}}
$$

and for any $k \geq 2$,

$$
\Gamma_{k k}^{1}=-\frac{u u_{x}}{1+u_{x}^{2}}, \quad \Gamma_{1 k}^{k}=\Gamma_{k 1}^{k}=\frac{u_{x}}{u} .
$$

Proof The required formulae follow by direct computation using

$$
\Gamma_{i j}^{k}=\frac{1}{2} g^{k l}\left(\bar{\nabla}_{i} g_{j l}+\bar{\nabla}_{j} g_{i l}-\bar{\nabla}_{l} g_{i j}\right)
$$

and the facts that

$$
\bar{\nabla}_{1} g_{i j}=\frac{\partial}{\partial x_{1}} g_{i j}= \begin{cases}2 u_{x} u_{x x} & i=j=1 \\ 2 u u_{x} \bar{\sigma}_{i j}, & i, j \geq 2 \\ 0 & \text { otherwise }\end{cases}
$$

and for $k \geq 2, \bar{\nabla}_{k} g_{i j}=0$.

Lemma 3.2. The nonzero components of $\nabla \mathcal{W}$ for axially symmetric hypersurfaces of the form (2) are

$$
\nabla_{1} h_{1}^{1}=\frac{-u_{x x x}}{\left(1+u_{x}^{2}\right)^{\frac{3}{2}}}+\frac{3 u_{x} u_{x x}^{2}}{\left(1+u_{x}^{2}\right)^{\frac{5}{2}}}
$$


and

$$
\nabla_{1} h_{k}^{k}=\frac{-u_{x}}{u^{2} \sqrt{1+u_{x}^{2}}}-\frac{u_{x} u_{x x}}{u\left(1+u_{x}^{2}\right)^{\frac{3}{2}}}=\frac{u_{x}}{u}\left(\kappa_{1}-\kappa_{2}\right) .
$$

Proof We use the formula

$$
\nabla_{i} h_{k}^{j}=\bar{\nabla}_{i} h_{k}^{j}+\Gamma_{i l}^{j} h_{k}^{l}-\Gamma_{i k}^{l} h_{l}^{j},
$$

the expressions for $h^{j}{ }_{k}$ from (3) and Lemma 3.1.

\section{Elementary flow behaviour}

We begin with an axially symmetric hypersurface given in the form (2) with a positive and at least twice differentiable initial graph function $u_{0}:[0, a] \rightarrow \mathbb{R}$ for which $F\left(\mathcal{W}_{0}\right)>0$, that is, the speed function is everywhere positive on $M_{0}$. We now deform the hypersurface such that the evolving graph function $u(x, t)$ describes an axially symmetric hypersurface flowing with speed in the normal direction equal to

$$
\frac{\partial X}{\partial t}=-F(\mathcal{W}) \nu
$$

where $F$ satisfies Conditions 2.1 and we prescribe pure Neumann boundary conditions at $x=0$ and $x=a$, that is,

$$
u_{x}(0, t)=u_{x}(a, t)=0
$$

for all $t$. As in [18], we add a tangential term to the normal speed such that the flow is well-defined; the equivalent evolution of the graph function $u(x, t)$ in the vertical direction is then

$$
\frac{\partial u}{\partial t}=-\sqrt{1+u_{x}^{2}} F(\mathcal{W})
$$

In view of (3) and Conditions 2.1, (iii), Eq. (6) can be rewritten as

$$
\frac{\partial u}{\partial t}=\frac{\dot{f}^{1}}{1+u_{x}^{2}} u_{x x}-\frac{(n-1) \dot{f}^{2}}{u} .
$$

Note in particular that since the matrix of the Weingarten map is everywhere diagonal, so is the matrix for $\dot{F}$, specifically

$$
\dot{F}^{i j}=\dot{f}^{i} \delta^{i j} .
$$

Details of this equivalence in coordinates which diagonalise the Weingarten map may be found in [4]. Moreover, in view of symmetry,

$$
\dot{f}^{2}\left(\kappa_{1}, \kappa_{2}, \ldots, \kappa_{2}\right)=\cdots=\dot{f}^{n}\left(\kappa_{1}, \kappa_{2}, \ldots, \kappa_{2}\right) ;
$$

here and throughout we will write $\dot{f}^{2}$.

Conditions 2.1, (ii) ensures existence at least for a short time of a solution to (7) with initial condition $u(\cdot, 0)=u_{0}$ and pure Neumann boundary conditions; we refer the reader to [18] for a discussion of short-time existence for solutions to the flow in this setting. 
Whereas in [18] evolution equations were mainly on $[0, a] \times \mathbb{S}^{n-1}$, here we use mainly equations on the evolving hypersurface. Specifically, we have the following evolution equations.

Lemma 4.1. Under the flow (6),

(i) $\frac{\partial}{\partial t} F=\mathcal{L} F+\dot{F}^{k l} h_{k}^{m} h_{m l} F$,

(ii) $\frac{\partial}{\partial t} H=\mathcal{L} H+\ddot{F}^{k l, r s} \nabla^{i} h_{k l} \nabla_{i} h_{r s}+\dot{F}^{k l} h_{k}^{m} h_{m l} H$,

(iii) $\frac{\partial}{\partial t} \kappa_{2}=\mathcal{L} \kappa_{2}+\dot{F}^{k l} h_{k m} h^{m}{ }_{l} \kappa_{2}+2 \dot{F}^{11} q^{2}\left(\kappa_{1}-\kappa_{2}\right)$,

where $\mathcal{L}=\dot{F}^{k l} \nabla_{k} \nabla_{l}$ and $\nabla$ denotes the covariant derivative on $M_{t}=X(\cdot, t)$.

Proof Equations (i) and (ii) are as in [2], for example, while (iii) is easily derived in the same way as in [14]. We note that we can actually compute the evolution of the individual principal curvatures because the Weingarten map is everywhere diagonal in our setting.

Because we have pure Neumann boundary conditions, we can reflect in the $x=0$ plane to create an even graph function which we then extend to a periodic solution of (6) on $\mathbb{R} \times[0, T)$. Then in applying the maximum principle we need only consider interior extrema. This idea was also used in $[10,14]$. Applications of the maximum principle to Lemma 4.1 lead to the following.

Corollary 4.2. Under the flow (6),

(i) If $F \geq 0$ everywhere on $M_{0}$, then $\min _{M_{t}} F \geq \min _{M_{0}} F$.

(ii) If $F$ is convex then if $H \geq 0$ everywhere on $M_{0}$, then $\min _{M_{t}} H \geq \min _{M_{0}} H$.

Proof These are direct applications of the maximum principle since Conditions 2.1 (ii) implies

$$
\dot{F}^{k l} h_{k m} h_{l}^{m}=\dot{f}^{1} \kappa_{1}^{2}+(n-1) \dot{f}^{2} \kappa_{2}^{2} \geq 0 .
$$

For part (ii), convexity of $F$ ensures that the gradient term has the correct sign.

\section{Remarks}

(i) As a consequence of our pinching estimate in Sect. 5, we will see that in fact $H>0$ remains true under (6) even if $F$ is not convex.

(ii) The Euler identity gives

$$
f=\dot{f}^{1} \kappa_{1}+\dot{f}^{2} \kappa_{2}+\ldots+\dot{f}^{n} \kappa_{n}=\dot{f}^{1} \kappa_{1}+(n-1) \dot{f}^{2} \kappa_{2},
$$

so in view of Conditions 2.1, (ii), since $F$ remains positive we must have everywhere at least one of the principal curvatures is positive and therefore $|A|>0$ holds under the flow.

Moreover, the evolution equation for $\kappa_{2}$ provides directly a uniform lower bound.

Proposition 4.3. Under the flow (6), the minimum of the rotational curvatures $\kappa_{2}$ does not decrease in time, that is

$$
\min _{M_{t}} \kappa_{2} \geq \min _{M_{0}} \kappa_{2}:=c_{0}>0
$$


Proof Since $q=-u_{x} \kappa_{2}$, we may rewrite Lemma 4.1, (iii) as

$$
\frac{\partial}{\partial t} \kappa_{2}=\mathcal{L} \kappa_{2}+\dot{F}^{k l} h_{k m} h_{l}^{m} \kappa_{2}+2 \dot{F}^{11} u_{x}^{2} \kappa_{2}^{2}\left(\kappa_{1}-\kappa_{2}\right) .
$$

Using Lemma 3.2, this can be rewritten as

$$
\frac{\partial}{\partial t} \kappa_{2}=\mathcal{L} \kappa_{2}+\dot{F}^{k l} h_{k m} h_{l}^{m} \kappa_{2}+2 \dot{F}^{11} u\left(\frac{u_{x}}{1+u_{x}^{2}}\right) \nabla_{1} \kappa_{2} .
$$

As in (8), the zero order term is nonnegative. Also, the coefficient of $\nabla_{1} \kappa_{2}$ is bounded, so the minimum of $\kappa_{2}$ does not decrease under the flow, by the maximum principle.

Remark In view of (3), Proposition 4.3 implies

$$
\sqrt{1+u_{x}^{2}} \leq \frac{1}{c_{0} u},
$$

that is, while $u>0$ the gradient $u_{x}$ remains bounded. We will provide an analogous bound on $u_{x x}$ in Sect. 5 .

\section{The pinching estimate}

We first characterise the gradient terms at extrema of degree zero homogeneous functions of the curvatures evolving under (6). A similar result was established in [6] for surfaces.

Lemma 5.1. Let $G(\mathcal{W})=g(\kappa(\mathcal{W}))$ be a smooth, symmetric, homogeneous of degree zero function in the principal curvatures of the axially symmetric hypersurface given by (2). At any stationary point of $G$ for which $\dot{G}$ is nondegenerate,

$$
\left(\dot{G}^{i j} \ddot{F}^{k l, r s}-\dot{F}^{i j} \ddot{G}^{k l, r s}\right) \nabla_{i} h_{k l} \nabla_{j} h_{r s}=\frac{2 f \dot{g}^{1}}{\kappa_{2}\left(\kappa_{2}-\kappa_{1}\right)}\left(\nabla_{1} h_{22}\right)^{2} .
$$

Proof It follows by a short contradiction argument, as in [6], that wherever $\dot{G}$ is nondegenerate we have that $\kappa_{1}, \kappa_{2} \neq 0$ and $\kappa_{2} \neq \kappa_{1}$. Using orthonormal coordinates at a stationary point of $G$, we have using (1) and Lemma 3.2 that

$$
\begin{aligned}
\dot{G}^{i j} \ddot{F}^{k l, r s} \nabla_{i} h_{k l} \nabla_{j} h_{r s}= & \dot{g}^{1}\left\{\ddot{f}^{11}\left(\nabla_{1} h_{11}\right)^{2}+2(n-1) \ddot{f}^{12} \nabla_{1} h_{11} \nabla_{1} h_{22}\right. \\
& \left.+(n-1)^{2} \ddot{f}^{22}\left(\nabla_{1} h_{22}\right)^{2}\right\} \\
& +2(n-1) \dot{g}^{2}\left(\frac{\dot{f}^{1}-\dot{f}^{2}}{\kappa_{1}-\kappa_{2}}\right)\left(\nabla_{1} h_{22}\right)^{2}
\end{aligned}
$$

and similarly

$$
\begin{aligned}
\dot{F}^{i j} \ddot{G}^{k l, r s} \nabla_{i} h_{k l} \nabla_{j} h_{r s}= & \dot{f}^{1}\left\{\ddot{g}^{11}\left(\nabla_{1} h_{11}\right)^{2}+2(n-1) \ddot{g}^{12} \nabla_{1} h_{11} \nabla_{1} h_{22}\right. \\
& \left.+(n-1)^{2} \ddot{g}^{22}\left(\nabla_{1} h_{22}\right)^{2}\right\} \\
& +2(n-1) \dot{f}^{2}\left(\frac{\dot{g}^{1}-\dot{g}^{2}}{\kappa_{1}-\kappa_{2}}\right)\left(\nabla_{1} h_{22}\right)^{2},
\end{aligned}
$$


SO

$$
\begin{aligned}
& \left(\dot{G}^{i j} \ddot{F}^{k l, r s}-\dot{F}^{i j} \ddot{G}^{k l, r s}\right) \nabla_{i} h_{k l} \nabla_{j} h_{r s} \\
& =\left(\dot{g}^{1} \ddot{f}^{11}-\dot{f}^{1} \ddot{g}^{11}\right)\left(\nabla_{1} h_{11}\right)^{2}+2(n-1)\left(\dot{g}^{1} \ddot{f}^{12}-\dot{f}^{1} \ddot{g}^{12}\right) \nabla_{1} h_{11} \nabla_{1} h_{22} \\
& \quad+\left[(n-1)^{2}\left(\dot{g}^{1} \ddot{f}^{22}-\dot{f}^{1} \ddot{g}^{22}\right)+\frac{2(n-1)\left(\dot{g}^{2} \dot{f}^{1}-\dot{f}^{2} \dot{g}^{1}\right)}{\kappa_{1}-\kappa_{2}}\right]\left(\nabla_{1} h_{22}\right)^{2} .
\end{aligned}
$$

Now

$$
\nabla_{1} G=\dot{g}^{1} \nabla_{1} h_{11}+(n-1) \dot{g}^{2} \nabla_{1} h_{22}
$$

so, since $G$ is nondegenerate,

$$
\nabla_{1} h_{11}=\frac{1}{\dot{g}^{1}}\left[\nabla_{1} G-(n-1) \dot{g}^{2} \nabla_{1} h_{22}\right]
$$

and (9) becomes

$$
\begin{aligned}
& \left(\dot{G}^{i j} \ddot{F}^{k l, r s}-\dot{F}^{i j} \ddot{G}^{k l, r s}\right) \nabla_{i} h_{k l} \nabla_{j} h_{r s} \\
& =\frac{\left(\dot{g}^{1} \ddot{f}^{11}-\dot{f}^{1} \ddot{g}^{11}\right)}{\left(\dot{g}^{1}\right)^{2}}\left(\nabla_{1} G\right)^{2} \\
& \quad+\frac{2(n-1)}{\dot{g}^{1}}\left[\left(\dot{g}^{1} \ddot{f}^{12}-\dot{f}^{1} \ddot{g}^{12}\right)-\frac{\dot{g}^{2}}{\dot{g}^{1}}\left(\dot{g}^{1} \ddot{f}^{11}-\dot{f}^{1} \ddot{g}^{11}\right)\right] \nabla_{1} h_{22} \nabla_{1} G \\
& \quad+(n-1)^{2}\left[\left(\frac{\dot{g}^{2}}{\dot{g}^{1}}\right)^{2}\left(\dot{g}^{1} \ddot{f}^{11}-\dot{f}^{1} \ddot{g}^{11}\right)-2 \frac{\dot{g}^{2}}{\dot{g}^{1}}\left(\dot{g}^{1} \ddot{f}^{12}-\dot{f}^{1} \ddot{g}^{12}\right)\right]\left(\nabla_{1} h_{22}\right)^{2} \\
& \quad+\left[(n-1)^{2}\left(\dot{g}^{1} \ddot{f}^{22}-\dot{f}^{1} \ddot{g}^{22}\right)+\frac{2(n-1)\left(\dot{g}^{2} \dot{f}^{1}-\dot{f}^{2} \dot{g}^{1}\right)}{\kappa_{1}}\right]\left(\nabla_{1} h_{22}\right)^{2}
\end{aligned}
$$

Since $g$ is homogeneous of degree zero, the Euler identity gives

$$
\dot{g}^{1} \kappa_{1}+(n-1) \dot{g}^{2} \kappa_{2}=0
$$

so, since $G$ is nondegenerate we may write

$$
\frac{\dot{g}^{2}}{\dot{g}^{1}}=-\frac{\kappa_{1}}{(n-1) \kappa_{2}}
$$

and the coefficient of $\left(\nabla_{1} h_{22}\right)^{2}$ in (10) becomes

$$
\begin{aligned}
& \frac{\dot{g}^{1}}{\kappa_{2}^{2}}\left[\ddot{f}^{11} \kappa_{1}^{2}+2(n-1) \ddot{f}^{12} \kappa_{1} \kappa_{2}+(n-1)^{2} \ddot{f}^{22} \kappa_{2}^{2}\right] \\
& -\frac{\dot{f}^{1}}{\kappa_{2}^{2}}\left[\ddot{g}^{11} \kappa_{1}^{2}+2(n-1) \ddot{g}^{12} \kappa_{1} \kappa_{2}+(n-1)^{2} \ddot{g}^{22} \kappa_{2}^{2}\right] \\
& +\frac{2(n-1) \dot{g}^{1}}{\kappa_{1}-\kappa_{2}}\left[-\frac{\kappa_{1}}{(n-1) \kappa_{2}} \dot{f}^{1}-\dot{f}^{2}\right] .
\end{aligned}
$$


Since $f$ is homogeneous of degree 1 , the first line above is identically equal to zero, while since $g$ is homogeneous of degree 0 , the first square bracketed term on the second line above is also identically equal to zero. The remaining term is equal to

$$
-\frac{2 \dot{g}^{1}}{\left(\kappa_{1}-\kappa_{2}\right) \kappa_{2}}\left[\dot{f}^{1} \kappa_{1}+(n-1) \dot{f}^{2} \kappa_{2}\right]=-\frac{2 f \dot{g}^{1}}{\left(\kappa_{1}-\kappa_{2}\right) \kappa_{2}}
$$

and we conclude that at an extremum of $G$,

$$
\left(\dot{G}^{i j} \ddot{F}^{k l, r s}-\dot{F}^{i j} \ddot{G}^{k l, r s}\right) \nabla_{i} h_{k l} \nabla_{j} h_{r s}=\frac{2 f \dot{g}^{1}}{\kappa_{2}\left(\kappa_{2}-\kappa_{1}\right)}\left(\nabla_{1} h_{22}\right)^{2}
$$

Theorem 5.2. Under the flow (5),

$$
H(x, t) \geq c_{1}|A(x, t)|,
$$

where $c_{1}=\min \left(\min _{[0, a]} \frac{H}{|A|}(\cdot, 0), 1\right)$. In particular, if $M_{0}$ has positive mean curvature, then $H>0$ continues to hold under the flow.

Proof The function $G=\frac{H}{|A|}$ is homogeneous of degree zero, so it evolves under (5) according to

$$
\frac{\partial}{\partial t} G=\mathcal{L} G+\left(\dot{G}^{i j} \ddot{F}^{k l, r s}-\dot{F}^{i j} \ddot{G}^{k l, r s}\right) \nabla_{i} h_{k l} \nabla_{j} h_{r s}
$$

(see, for example, [6]). We have $g\left(\kappa_{1}, \ldots, \kappa_{n}\right)=\frac{\kappa_{1}+\ldots+\kappa_{n}}{\sqrt{\kappa_{1}^{2}+\ldots+\kappa_{n}^{2}}}$ so

$$
\begin{aligned}
& \dot{g}^{1}\left(\kappa_{1}, \kappa_{2}, \ldots, \kappa_{2}\right)=\frac{(n-1) \kappa_{2}\left(\kappa_{2}-\kappa_{1}\right)}{|A|^{3}} \text { and } \\
& \dot{g}^{2}\left(\kappa_{1}, \kappa_{2}, \ldots, \kappa_{2}\right)=\frac{(n-1) \kappa_{1}\left(\kappa_{1}-\kappa_{2}\right)}{|A|^{3}} .
\end{aligned}
$$

Suppose now we are at a spatial minimum of $G$. At this point, $\dot{G}$ could be nondegenerate or degenerate. If $\dot{G}$ is nondegenerate, then using Lemma 5.1 we have

$$
\left(\dot{G}^{i j} \ddot{F}^{k l, r s}-\dot{F}^{i j} \ddot{G}^{k l, r s}\right) \nabla_{i} h_{k l} \nabla_{j} h_{r s}=\frac{2(n-1) f}{|A|^{3}}\left(\nabla_{1} h_{22}\right)^{2}>0
$$

and the maximum principle applied to (11) gives that the minimum of $G$ does not decrease. On the other hand, if $\dot{G}$ is degenerate, then from (12) either $\kappa_{1}=0$, or $\kappa_{1}=\kappa_{2}$ (the case $\kappa_{2}=0$ does not occur in view of Proposition 4.3). It follows from the Euler identity and Corollary 4.2, (i) that wherever $\kappa_{1}=\kappa_{2}$, the principal curvatures are positive.

- If $\kappa_{1}=0$ then

$$
g\left(0, \kappa_{2}\right)=\frac{(n-1) \kappa_{2}}{\sqrt{(n-1) \kappa_{2}^{2}}}=\sqrt{n-1},
$$

a positive lower bound on $G$. 
- If $\kappa_{1}=\kappa_{2}$ then

$$
g\left(\kappa_{2}, \kappa_{2}\right)=\frac{n \kappa_{2}}{\sqrt{n \kappa_{2}^{2}}}=\sqrt{n},
$$

so $g$ achieves its absolute maximum, namely the equality case of the Cauchy-Schwarz inequality, at the supposed minimum. Thus $g$ must be identically constant and $M_{t}$ is umbilic, which is impossible.

It follows that $G$ is bounded below by $c_{1}:=\min \left(\min _{M_{0}} G, 1\right)$. In the case that $M_{0}$ has positive mean curvature, $c_{1}>0$ and the second statement of the lemma follows.

Remarks

1. Theorem 5.2 and the Cauchy-Schwarz inequality imply

$$
0<c_{0} \leq \frac{H}{|A|} \leq \frac{1}{\sqrt{n}}
$$

under (5). If we restrict to $\{\kappa \in \Gamma:|A|=1\}$, the curvatures remain within a compact subset. Since $\dot{f}$ is continuous and homogeneous of degree zero, this in turn implies (5) is uniformly parabolic; for each $i$

$$
\underline{C} \leq \dot{f}^{i} \leq \bar{C}
$$

is maintained under the flow.

2. By the same argument as in 1. above, any homogeneous of degree zero function of the principal curvatures is bounded above and below under the flow. In particular, considering the function $\frac{H}{F}$ we have

$$
F \geq \underline{c}_{0} H \text {. }
$$

Similarly trace $\dot{F}=\sum_{i=1}^{n} \dot{f}^{i}$ is another homogeneous of degree zero function, so under the flow

$$
\sum_{i=1}^{n} \dot{f}^{i} \leq \bar{c}_{0}
$$

(In the case that $F$ is convex, it follows algebraically that one may take $\underline{c}_{0}=\frac{1}{n}$ and $\bar{c}_{0}=1$ in the above two inequalities.) These inequalities were critical in the analysis in [18]; in the case of pure Neumann boundary conditions they may be replaced by the above inequalities such that the results of that paper carry over for $F$ homogeneous of degree 1 and not necessarily convex. Specifically we have the following partial singularity characterisation:

Theorem 5.3. Let $M_{0}$ be an axially symmetric hypersurface given by (2) for some positive, nondecreasing function $u_{0} \in C^{2}([0, a])$. Suppose $F$ satisfies Conditions 2.1 and is everywhere nonnegative on $M_{0}$. There exists a unique solution $u \in C^{2}([0, a] \times[0, T)), T<\infty$ to (6) with pure Neumann boundary conditions. If, additionally,

$$
\lim _{z \rightarrow-\infty} f(z, 1, \ldots, 1)<0
$$


where we allow the case that the limit is equal to $-\infty$, then if $\lim _{t \rightarrow T} \kappa_{1}^{2}(a, t)<$ $\infty$ we have

$$
\lim _{t \rightarrow T} \kappa_{j}^{2}(0, t)=\infty
$$

for $j=2, \ldots, n$.

Together with some additional arguments, the pinching estimate of Theorem 5.2 may also be used to show the ratio $\frac{\kappa_{1}}{\kappa_{2}}$ remains bounded under (5).

Corollary 5.4. Under the flow (6), the ratio $\frac{\kappa_{1}^{2}}{\kappa_{2}^{2}}$ remains bounded.

Proof We will prove this result by considering three cases separately. We know from Theorem 5.2 that $H>0$ continues to hold under the flow, so

$$
\kappa_{1}>-(n-1) \kappa_{2}
$$

(in fact, a slightly stronger statement involving $c_{1}$ is possible from Theorem $5.2)$ and if $\kappa_{1}<0$, then we have

$$
\kappa_{1}^{2} \leq(n-1)^{2} \kappa_{2}^{2}
$$

If, instead, $0 \leq \kappa_{1} \leq c_{0}$, then from Proposition 4.3 we have

$$
0<\kappa_{1} \leq \kappa_{2}
$$

and so

$$
\kappa_{1}^{2} \leq \kappa_{2}^{2} \leq(n-1)^{2} \kappa_{2}^{2} .
$$

Finally, in the case $\kappa_{1} \geq c_{0}$ we have

$$
2(n-1) \kappa_{1} \kappa_{2} \geq 2 \kappa_{1} \kappa_{2} \geq 2 c_{0}^{2}
$$

and

$$
H^{2}-|A|^{2}=2(n-1) \kappa_{1} \kappa_{2}+(n-1)(n-2) \kappa_{2}^{2} \geq 2(n-1) \kappa_{1} \kappa_{2}
$$

SO

$$
\frac{H^{2}}{|A|^{2}} \geq 1+\frac{2(n-1) \kappa_{1} \kappa_{2}}{|A|^{2}} \geq 1+\varepsilon
$$

for some $\varepsilon>0$, since the homogeneous of degree zero function $\frac{\kappa_{1} \kappa_{2}}{|A|^{2}}$ attains a positive minimum on the set $\left\{\kappa=\left(\kappa_{1}, \kappa_{2}\right):|A|=2 c_{0}, \kappa_{1}, \kappa_{2} \geq c_{0}\right\}$.

Therefore

$$
\frac{\left[\kappa_{1}+(n-1) \kappa_{2}\right]^{2}}{\kappa_{1}^{2}+(n-1) \kappa_{2}^{2}} \geq 1+\varepsilon
$$

SO

$$
\kappa_{1}^{2}+2(n-1) \kappa_{1} \kappa_{2}+(n-1)^{2} \kappa_{2}^{2} \geq(1+\varepsilon)\left[\kappa_{1}^{2}+(n-1) \kappa_{2}^{2}\right]
$$

In other words,

$$
\begin{aligned}
\varepsilon \kappa_{1}^{2} & \leq(n-1)(n-2-\varepsilon) \kappa_{2}^{2}+2(n-1) \kappa_{1} \kappa_{2} \\
& \leq(n-1)(n-2-\varepsilon) \kappa_{2}^{2}+\eta \kappa_{1}^{2}+\frac{1}{4 \eta} \kappa_{2}^{2}
\end{aligned}
$$


for any $\eta>0$. Choosing $\eta=\frac{\varepsilon}{2}$ gives

$$
\kappa_{1}^{2} \leq \frac{2}{\varepsilon}(n-1)\left(n-2-\varepsilon+\frac{1}{2 \varepsilon}\right) \kappa_{2}^{2}
$$

We have shown that in all cases $\kappa_{1}^{2}$ is bounded by $\kappa_{2}^{2}$, by a constant depending only on $n$ and $M_{0}$. This completes the proof.

Corollary 5.5. Under the flow (6), there exists a constant $C$, depending only on $n$ and $M_{0}$ such that

$$
\left(u_{x x}\right)^{2} \leq \frac{C}{u^{4}}
$$

Proof From (4) and Theorem 5.2 we have that under the flow,

$$
\left(u_{x x}\right)^{2} \leq C \frac{\left[1+u_{x}^{2}\right]^{2}}{u^{2}}
$$

is preserved. The result follows in view of the remark after Proposition 4.3 .

\section{The singularity}

Given an initial hypersurface $M_{0}$ as in (2), comparison with an enclosing cylinder also flowing under (5) shows that the maximal existence time $T$ of a solution to (5) with initial hypersurface $M_{0}$ is finite. Moreover, as $t \rightarrow T$ we must have $u \rightarrow 0$, that is, the evolving hypersurface approaches the axis of rotation, because if not, then $u>0$ at time $T$ and then Proposition 4.3 and Corollary 5.5 imply $u_{x}$ and $u_{x x}$ are bounded, so $M_{T}$ is a $C^{2}$ hypersurface which could be used in the short time existence result, contradicting the maximality of $T$. Therefore, there is some $x \in[0, a]$ such that $|A|^{2}(x, t) \rightarrow \infty$ and $u(x, t) \rightarrow 0$ as $t \rightarrow T$.

Here we characterise the curvature singularity of an axially symmetric hypersurface with positive $F$ evolving under (5) as Type I, analogous to the case of evolution of axially symmetric surfaces of positive mean curvature by the mean curvature flow [14].

Let $F_{0}:=\min _{M_{0}} F$. In view of uniform parabolicity, a short argument (Lemma 2.5 in [6]) shows that, under the flow (5),

$$
F(\mathcal{W}(x, t)) \geq \frac{F_{0}}{\sqrt{1-2 \underline{C} F_{0}^{2} t}}
$$

In analogy with the case of mean curvature flow [14], we say a curvature singularity is Type I if there is a $C>0$ such that

$$
\lim _{t \rightarrow T} \max _{M_{t}}|A| \leq \frac{C}{\sqrt{T-t}} .
$$

If the blow-up rate of $|A|$ is faster than the above right hand side the curvature singularity is said to be Type II.

Example The blow-up rate of cylinders is Type I. 

becomes

In the case of a cylinder, say $\kappa_{1}=0, \kappa_{2}=\ldots=\kappa_{n}=\frac{1}{r}, u_{x} \equiv 0$ and (6)

$$
\frac{d r}{d t}=-f\left(0, \frac{1}{r}, \ldots, \frac{1}{r}\right)=-\frac{f_{0}}{r}
$$

where $f_{0}:=f(0,1, \ldots, 1)$. If the cylinder shrinks to a line at time $T$ then

$$
r(t)=\sqrt{2 f_{0}(T-t)}
$$

and the curvature evolves according to

$$
|A|=\frac{n-1}{\sqrt{2 f_{0}(T-t)}}
$$

so the singularity is Type I.

Theorem 6.1. Let $M_{0}$ be an axially symmetric hypersurface given by (2) for some positive function $u_{0} \in C^{2}([0, a])$. Suppose $F$ satisfies Conditions 2.1 and is everywhere strictly positive on $M_{0}$. If $T$ is the maximal existence time then the norm of the second fundamental form satisfies

$$
\max _{M_{t}}|A|^{2} \leq \frac{C}{T-t}
$$

for all $t<T$.

Proof We will use a modification of the argument as in Theorem 5.7 of [1] taking into account our more general flow speed $F$. In view of preserved curvature pinching, Theorem 5.2, there is a positive constant $C$ such that

$$
\frac{\dot{F}^{k p} \dot{F}_{p l} h_{k}^{m} h_{m l}}{F^{2}} \leq C^{2}
$$

since the quantity on the left hand side is homogeneous of degree zero in the principal curvatures. This means precisely that

$$
\frac{\left(\dot{f}^{1}\right)^{2} u_{x x}^{2}}{\left(1+u_{x}^{2}\right)^{3}}+\frac{(n-1)\left(\dot{f}^{2}\right)^{2}}{u^{2}\left(1+u_{x}^{2}\right)} \leq C^{2}\left[\frac{(n-1) \dot{f}^{2}}{u \sqrt{1+u_{x}^{2}}}-\frac{\dot{f}^{1} u_{x x}}{\left(1+u_{x}^{2}\right)^{\frac{3}{2}}}\right]^{2}
$$

where the quantity inside the brackets on the right hand side, namely $F$, is strictly positive by Lemma 4.1, (i). Neglecting the second term on the left hand side, it follows that

$$
\frac{\dot{f}^{1} u_{x x}}{1+u_{x}^{2}} \leq C\left[\frac{(n-1) \dot{f}^{2}}{u}-\frac{\dot{f}^{1} u_{x x}}{1+u_{x}^{2}}\right]
$$

and therefore

$$
\frac{\dot{f}^{1} u_{x x}}{1+u_{x}^{2}} \leq \frac{C}{C+1} \frac{(n-1) \dot{f}^{2}}{u}
$$


Using (7) we estimate

$$
\begin{aligned}
\frac{\partial u}{\partial t}= & \frac{\dot{f}^{1}}{1+u_{x}^{2}} u_{x x}-\frac{(n-1) \dot{f}^{2}}{u} \leq\left[\frac{C}{C+1}-1\right] \frac{(n-1) \dot{f}^{2}}{u} \\
= & \frac{-1}{C+1} \frac{(n-1) \dot{f}^{2}}{u} \leq \frac{-(n-1) \underline{C}}{C+1} \frac{1}{u}=:-\frac{\delta}{u}
\end{aligned}
$$

where we have used (13).

Now fix $x$ and integrate:

$$
\int_{t}^{T} \frac{\partial}{\partial \tau} \frac{1}{2} u^{2}(x, \tau) d \tau \leq-\delta(T-t)
$$

this implies

$$
u^{2}(x, t) \geq u^{2}(x, T)+2 \delta(T-t) .
$$

It follows that

$$
\kappa_{2}^{2}(x, t) \leq \frac{1}{u^{2}(x, t)} \leq \frac{1}{u^{2}(x, T)+2 \delta(T-t)} \leq \frac{1}{2 \delta(T-t)}
$$

and in view of Corollary 5.4

$$
\kappa_{1}^{2}(x, t) \leq C^{2} \kappa_{2}^{2}(x, t) \leq \frac{C^{2}}{2 \delta(T-t)} .
$$

The result follows.

\section{Closed, axially symmetric hypersurfaces}

In this section we turn our attention to closed, convex, axially symmetric hypersurfaces without boundary evolving under (5). There has been much previous work on closed convex hypersurfaces contracting under flows such as (5), without the condition of axial symmetry, beginning with the famous result of Huisken for the mean curvature flow [13]. We refer the reader to [9] for a discussion on previous work for fully nonlinear speeds satisfying natural conditions such as Conditions 2.1. Crucial in most of the previous work is a second order condition on the speed such as convexity, or concavity together with another natural condition either on the speed or on the initial hypersurface, the latter in the form of a curvature pinching condition. Andrews has covered many cases $[2,4]$; these and further generalisations are discussed in [9]. Andrews and the first author were able to show contraction to round points without a second order condition for hypersurfaces sufficiently close to spheres in [8], that is, for hypersurfaces already very strongly curvature pinched. In the special case of contracting surfaces, that is, $n=2$, no second order condition on the speed, nor any initial curvature pinching is needed [5]. In this section, we extend Andrews' result for surfaces to the case of axially symmetric hypersurfaces, again without any second order condition on the speed. 
Theorem 7.1. Let $M_{0}$ be a closed, smooth, strictly convex, axially symmetric $n$-dimensional hypersurface without boundary, $n \geq 2$ smoothly embedded in $\mathbb{R}^{n+1}$ by $X_{0}: \mathbb{S}^{n} \rightarrow \mathbb{R}^{n+1}$. Let $F$ satisfy Conditions 2.1. Then there exists a unique family of smooth, strictly convex, axially symmetric hypersurfaces $\left\{M_{t}=X_{t}\left(\mathbb{S}^{n}\right)\right\}_{0<t<T}$ satisfying (5), with initial condition $X(x, 0)=X_{0}(x)$ for all $x \in \mathbb{S}^{n}$. The solution exists on a finite maximal time interval $[0, T)$ and the image converges uniformly to a point $p \in \mathbb{R}^{n+1}$ as $t \rightarrow T$. The rescaled maps $\frac{X_{t}-p}{\sqrt{2(T-t)}}$ converge smoothly and exponentially to an embedding $\tilde{X}_{T}$ whose image is equal to the unit sphere in $\mathbb{R}^{n+1}$ centred at the origin.

Proof The argument to obtain curvature pinching, in this setting positive bounds above and below on the ratio $\frac{\kappa_{2}}{\kappa_{1}}$ of the two potentially different principal curvatures, is very similar to that presented in [5], so we just point out the necessary adjustments. A suitable pinching function here is the natural generalisation of that in [5], namely

$$
G(\mathcal{W})=\frac{n\left|A^{0}\right|^{2}}{H^{2}}
$$

where $\left|A^{0}\right|^{2}=|A|^{2}-\frac{1}{n} H^{2}$ is the trace-free norm of the second fundamental form, a natural pointwise measure for convex hypersurfaces of their closeness to a sphere. This function $G$ corresponds to

$$
g(\kappa(\mathcal{W}))=\frac{n\left(\kappa_{1}^{2}+\ldots+\kappa_{n}^{2}\right)-\left(\kappa_{1}+\ldots+\kappa_{n}\right)^{2}}{\left(\kappa_{1}+\ldots+\kappa_{n}\right)^{2}} .
$$

Denoting as earlier the curvature in the axially direction as $\kappa_{1}$, we have $\kappa_{2}=\ldots=\kappa_{n}$ and, by slight abuse of notation, may rewrite

$$
g\left(\kappa_{1}, \kappa_{2}\right)=\frac{n \kappa_{1}^{2}+n(n-1) \kappa_{2}^{2}-\left(\kappa_{1}+(n-1) \kappa_{2}\right)^{2}}{\left(\kappa_{1}+(n-1) \kappa_{2}\right)^{2}} .
$$

It is easy to compute that

$$
\dot{g}^{1}=\frac{2 n(n-1) \kappa_{2}\left(\kappa_{1}-\kappa_{2}\right)}{H^{3}} \text { and } \dot{g}^{2}=\frac{2 n \kappa_{1}\left(\kappa_{2}-\kappa_{1}\right)}{H^{3}} .
$$

We can show that the maximum of $G$ is not increasing in time: restricting ourselves initially to a short time interval on which $M_{t}$ remains convex, at a maximum point $\left(x_{0}, t_{0}\right)$ of $G, t_{0}>0$, we must have $\left.G\right|_{\left(x_{0}, t_{0}\right)}>0$ and therefore $\kappa_{1} \neq \kappa_{2}$ at that point since otherwise, $\left.G\right|_{\left(x, t_{0}\right)} \equiv 0$ so $\kappa_{1} \equiv \kappa_{2}$ and $M_{t_{0}}$ is a sphere. Therefore, $\dot{G}$ is nondegenerate at this maximum point and, in view of Lemma 5.1, we have that the gradient term in the evolution equation (11) for $G$, is equal to

$$
\frac{2 f \dot{g}^{1}}{\kappa_{2}\left(\kappa_{2}-\kappa_{1}\right)}\left(\nabla_{1} h_{22}\right)^{2}=-\frac{4 n(n-1) f}{H^{3}}\left(\nabla_{1} h_{22}\right)^{2}<0 .
$$

It follows by the maximum principle that the maximum of $G$ does not increase. 
At such a maximum point of $G$, there are two possibilities: $\kappa_{2}>\kappa_{1}$ or $\kappa_{1}>\kappa_{2}$. In the former case,

$$
1<r=\frac{\kappa_{2}}{\kappa_{1}}=\frac{1}{n-1}\left[\frac{n}{1-\sqrt{(n-1) G}}-1\right],
$$

so that $G$ does not increase implies $r$ does not increase. In the latter case

$$
1>r=\frac{\kappa_{2}}{\kappa_{1}}=\frac{1}{n-1}\left[\frac{n}{1+\sqrt{(n-1) G}}-1\right],
$$

so that $G$ does not increase implies $r$ does not decrease.

In other words, we have shown that the pinching ratio does not deviate further from 1 and so the ratio $\frac{\kappa_{2}}{\kappa_{1}}$ is bounded above and below by its initial extreme values. Pinching, together with the absolute lower bound on $F$ (analogous to Corollary 4.2, (i)) gives that the evolving hypersurface remains convex, so the above argument applies up to time $T$.

In view of curvature pinching, the proof of Theorem 7.1 may be completed following the corresponding arguments in [8], see also the concluding remarks of that paper, since there no convexity condition on the speed was required.

Remark As in [5], there is a corresponding result if $F$ is instead homogeneous of degree $\alpha>1$, provided the initial hypersurface is sufficiently curvature pinched. Specifically, the proof proceeds as above, except that, in view of the homogeneity of $F$, the gradient term in the evolution equation for $G$ now becomes

$$
\frac{2 n \alpha(n-1) f}{H^{3}}\left[(\alpha-1) \frac{\kappa_{1}}{\kappa_{2}}-(1+\alpha)\right]\left(\nabla_{1} h_{22}\right)^{2} .
$$

For this to be nonpositive requires the pinching ratio of the principal curvatures to be not greater than

$$
\frac{\alpha+1}{\alpha-1}=1+\frac{2}{\alpha-1} .
$$

The pinching estimate can also be used to show that convex, axially symmetric hypersurfaces contracting self-similarly under (5) are necessarily spheres. This complements other results on compact self-similar hypersurfaces contracting under curvature flows, such as those in [14,17]. Such hypersurfaces satisfy the corresponding elliptic equation

$$
\langle X, \nu\rangle=F(\mathcal{W})
$$

and the characterisation as spheres may be deduced by considering the corresponding elliptic equation satisfied by the curvature pinching function.

Theorem 7.2. If $M$ is a closed, strictly convex, axially symmetric hypersurface satisfying (16), where $F(\mathcal{W})=f(\kappa)$ is positive, symmetric and homogeneous of degree 1 , then $M$ is a unit sphere. 
Proof In view of (16), the function $G$, as defined in (14), satisfies

$$
\mathcal{L} G=\left(\dot{F}^{i j} \ddot{G}^{k l, p q}-\dot{G}^{i j} \ddot{F}^{k l, p q}\right) \nabla_{i} h_{k l} \nabla_{j} h_{p q}+\langle X, \nabla G\rangle .
$$

Suppose that $G$ obtains a local maximum on $M$. At such a local maximum point of $G$, it must be that $G>0$ because otherwise $G \equiv 0$ and $M$ is a sphere. Therefore, $\dot{G}$ is nondegenerate at this maximum point, and, in view of Lemma 5.1 , we have

$$
\begin{aligned}
\left(\dot{F}^{i j} \ddot{G}^{k l, p q}-\dot{G}^{i j} \ddot{F}^{k l, p q}\right) \nabla_{i} h_{k l} \nabla_{j} h_{p q} & =-\frac{2 f \dot{g}^{1}}{\kappa_{2}\left(\kappa_{2}-\kappa_{1}\right)}\left(\nabla_{1} h_{22}\right)^{2} \\
& =\frac{4 n(n-1) f}{H^{3}}\left(\nabla_{1} h_{22}\right)^{2}>0,
\end{aligned}
$$

which is a contradiction to $G$ having a local maximum. Therefore $G$ must be identically constant, and if $M$ is not a sphere $G>0$ and everywhere on $M$ we have $\kappa_{1} \neq \kappa_{2}$. In this case, that the first term in (17) is identically equal to zero implies that $\nabla_{1} h_{22} \equiv 0$, and since $\nabla G \equiv 0$ we have

$$
0 \equiv \dot{g}^{1} \nabla_{1} h_{11}+(n-1) \dot{g}^{2} \nabla_{1} h_{22}
$$

so $\nabla_{1} h_{11} \equiv 0$ also because $\dot{g}^{1} \neq 0$. In view of Lemma 3.2 , it follows that $M$ is a sphere.

Remark Again there is a corresponding result for speeds $F$ homogeneous of degree $\alpha>1$ : if the closed, convex, axially symmetric hypersurface $M$ satisfies (16) and has curvature pinching ratio not greater than (15), then it must be a sphere.

\section{References}

[1] Altschuler, S., Angenent, S.B., Giga, Y.A.: Mean curvature flow through singularities for surfaces of rotation. J. Geom. Anal. 5(3), 293-358 (1995)

[2] Andrews, B.H.: Contraction of convex hypersurfaces in Euclidean space. Calc. Var. Partial Differ. Equ. 2(2), 151-171 (1994)

[3] Andrews, B.H.: Gauss curvature flow: the fate of the rolling stones. Invent. Math. 138(1), 151-161 (1999)

[4] Andrews, B.H.: Pinching estimates and motion of hypersurfaces by curvature functions. J. Reine Angew. Math. 608, 17-33 (2007)

[5] Andrews, B.H.: Moving surfaces by non-concave curvature functions. Calc. Var. Partial Differ. Equ. 39(3-4), 649-657 (2010)

[6] Andrews, B.H., Langford, M., McCoy, J.A.: Convexity estimates for fully nonlinear surface flows. J. Differ. Geom. (to appear)

[7] Andrews, B.H., Langford, M., McCoy, J.A.: Convexity estimates for hypersurfaces moving by convex curvature functions. Anal. PDE 7(2), 407-433 (2014) 
[8] Andrews, B.H., McCoy, J.A.: Convex hypersurfaces with pinched principal curvatures and flow of convex hypersurfaces by high powers of curvature. Trans. Am. Math. Soc. 364, 3427-3447 (2012)

[9] Andrews, B.H., McCoy, J.A., Zheng, Y.: Contracting convex hypersurfaces by curvature. Calc. Var. Partial Differ. Equ. 47(3-4), 611-665 (2013)

[10] Athanassenas, M.: Volume-preserving mean curvature flow of rotationally symmetric surfaces. Comment. Math. Helv. 72(1), 52-66 (1997)

[11] Dzuik, G., Kawohl, B.: On rotationally symmetric mean curvature flow. J. Differ. Equ. 93(1), 142-150 (1991)

[12] Escher, J., Matioc, B.-V.: Neck pinching for periodic mean curvature flows. Analysis 30, 253-260 (2010)

[13] Huisken, G.: Flow by mean curvature of convex surfaces into spheres. J. Differ. Geom. 20(1), 237-266 (1984)

[14] Huisken, G.: Asymptotic behaviour for singularities of the mean curvature flow. J. Differ. Geom. 31, 285-299 (1990)

[15] Matioc, B.-V.: Boundary value problems for rotationally symmetric mean curvature flows. Arch. Math. 89, 365-372 (2007)

[16] McCoy, J.A.: Mixed volume preserving curvature flows. Calc. Var. Partial Differ. Equ. 24, 131-154 (2005)

[17] McCoy, J.A.: Self-similar solutions of fully nonlinear curvature flows. Ann. Scuola Norm. Sup. Pisa Cl. Sci. 10(5), 317-333 (2011)

[18] McCoy, J.A., Mofarreh, F.Y.Y., Williams, G.H.: Fully nonlinear curvature flow of axially symmetric hypersurfaces with boundary conditions. Annali di Matematica Pura ed Applicata, 1-13 (2013, to appear in print). doi:10.1007/ s10231-013-0337-7

James A. McCoy, Fatemah Y. Y. Mofarreh and Valentina-Mira Wheeler

Institute for Mathematics and its Applications

University of Wollongong

Northfields Av

Wollongong

NSW 2522

Australia

e-mail: jamesm@uow.edu.au

Fatemah Y. Y. Mofarreh

e-mail: fyym102@uowmail.edu.au

Valentina-Mira Wheeler

e-mail: vwheeler@uow.edu.au

Received: 7 November 2013.

Accepted: 6 September 2014. 